\title{
EFECTO DE LA RADIACIÓN UV-C SOBRE LA CAPACIDAD ANTIOXIDANTE Y CONTENIDO DE FENOLES TOTALES EN FRUTOS EXÓTICOS DEL ECUADOR: NARANJILLA (Solanum quitoense) $Y$ MORTIÑO (Vaccinium floribundum)
}

Autores:

María José Andrade'

Carlota Moreno'

Analía Concellón ${ }^{2}$ 



\section{Resumen}

El objetivo del presente trabajo fue evaluar el efecto de la aplicación de la radiación UV-C sobre el contenido de compuestos antioxidantes y la capacidad antioxidante de la naranjilla y el mortiño, durante el almacenamiento refrigerado. Frutos recién cosechados fueron seleccionados y divididos en dos grupos: frutos tratados (irradiados con dosis de $12.5 \mathrm{~kJ} / \mathrm{m} 2$ ) y no tratados (controles) y almacenados en bandejas plásticas a $5^{\circ} \mathrm{C}$. A los $0,7,14,21$ y 28 días se determinó el contenido de flavonoides, fenoles totales, ácido ascórbico, carotenoides totales (naranjilla), antocianinas (mortiño) y la capacidad antioxidante.

En el caso de la naranjilla, inmediatamente después de la aplicación del tratamiento con radiación UV-C (12.5 kJ/m2) se observó una disminución significativa del contenido de carotenoides y de la capacidad antioxidante; no se produjeron diferencias en el contenido de flavonoides, ácido ascórbico y fenoles con respecto a las muestras control. A lo largo del almacenamiento, el contenido de flavonoides disminuyó en frutos tratados y controles. El contenido de fenoles se mantuvo constante hasta el día 7 para controles y hasta el día 14 para tratados, éstos últimos alcanzando en el día 28, mayor concentración que los controles. La concentración de ácido ascórbico se mantuvo constante hasta el día 21 en los frutos tratados, mientras que en los controles disminuyó a lo largo del almacenamiento. A partir del día 7 hasta el final del almacenamiento el contenido de carotenoides fue mayor en los frutos tratados que controles, encontrándose una reducción de $28 \%$ (controles) y $14 \%$ (tratados) respecto al día 0 . La mayor capacidad antioxidante en los frutos tratados se presentó en los días 7 y 28 ; en relación al día inicial, se encontró una disminución de 40\% y 32\% para frutos control y tratados, respectivamente. El tratamiento con radiación UV-C permitió mantener mayor concentración de carotenoides, fenoles y capacidad antioxidante a tiempos largos de almacenamiento, lo que probablemente podría estar relacionado con la calidad e incremento de la vida útil del fruto.

En el mortiño, inmediatamente después del tratamiento con radiación UV-C se observó mayor concentración de ácido ascórbico, fenoles totales y flavonoides. Durante el almacenamiento los frutos tratados y controles presentaron reducción del contenido de FLAV, FT y AsA. Los frutos tratados presentaron menor pérdida de ácido ascórbico. El tratamiento permitió mantener estable el contenido de antocianinas a lo largo del almacenamiento. La capacidad antioxidante de los frutos tratados (12,5 kJ/m2) se mantuvo constante durante 21 días.

Palabras clave: naranjilla, mortiño, radiación UV-C, poscosecha, antioxidantes.

Recibido: Julio 2012 


\section{Abstract}

The aim of this study was to evaluate the effect of the application of UV-C radiation on the content of antioxidant compounds and antioxidant capacity of naranjilla and mortiño, during refrigerated storage. Freshly harvested fruits were selected and divided into two groups: fruits treated (irradiated with doses of $12.5 \mathrm{~kJ} / \mathrm{m2}$ ) and untreated (control) and stored in plastic trays at $5^{\circ} \mathrm{C}$. At $0,7,14,21$ and 28 days was determined the content of flavonoids, total phenolics, ascorbic acid, total carotenoids (naranjilla), anthocyanins (mortiño) and antioxidant capacity.

For naranjilla, immediately after applying the treatment with UV-C radiation $(12.5 \mathrm{~kJ} / \mathrm{m} 2)$ showed a significant decrease of the content of carotenoids and antioxidant capacity, there were no differences in the content of flavonoids, ascorbic acid and phenol with respect to the control samples. During storage, reduced flavonoid content in fruit treated and controls. The phenol content remained constant until day 7 to controls and to day 14 for treated, the latter reaching in 28 days, greater concentration than controls. The ascorbic acid concentration was kept constant until day 21 in the treated fruit while in the controls decreased over storage. From day 7 until the end of storage carotenoid content was higher in treated fruit than controls, found a reduction of $28 \%$ (controls) and $14 \%$ (treated) compared to day 0 . The highest antioxidant capacity in fruits treated appeared on days 7 and 28 , in relation to the initial days, there was a decrease of $40 \%$ and $32 \%$ for control and treated fruits, respectively. The treatment with UV-C radiation highest concentration possible to maintain carotenoid, antioxidant phenols and long times of storage, which could possibly be related to the quality and increasing the life of the fruit.

In mortiño, immediately after treatment with UV-C showed higher concentration of ascorbic acid, total phenols and flavonoids. During storage and controls treated fruits showed FLAV content reduction, FT and AoA. The treated fruits had lower ascorbic acid loss. Treatment was allowed to maintain stable anthocyanin content during storage. The antioxidant capacity of fruits treated (12.5 kJ/m2) remained constant for 21 days.

Keywords: naranjilla, mortiño, UV-C, postharvest, antioxidants 


\section{Introducción}

La radiación UV es una radiación no ionizante, forma parte del espectro electromagnético, comprendida entre 100 a $400 \mathrm{~nm}$; se clasifica en tres tipos, UV-A (315-400 nm), UV-B (280-315 nm) y UV-C (200-280 nm). Ésta última tiene su pico máximo de emisión a 254 nm (Artés \& Allende, 2005). En función de la intensidad y longitud de onda la radiación UV-C puede inducir un estrés biológico en plantas y activar algunos mecanismos de defensa de los tejidos vegetales con la consecuente producción de fitoalexinas (Mercier, Arul, \& Julien, 1993).

Según Cisneros-Zevallos (2003), la aplicación poscosecha de un tipo de estrés abiótico controlado (exposición a luz UV-C) induce la producción e incremento de la síntesis de compuestos fotoquímicos con actividad nutracéutica, o la reducción de compuestos indeseables. Así el control del estrés inducido por la luz UV-C puede usarse como una herramienta para reforzar las propiedades benéficas de productos frescos enteros o cortados

La radiación UV-C se ha estudiado como un tratamiento alternativo o que combinado con la refrigeración contribuyen a la preservación de frutas y hortalizas, en el control de enfermedades poscosecha (Allende \& Artés, 2003; Maharaj, Azul, \& Nadeau, 1999), no deja residuos, no afecta las características sensoriales, sabor y aroma del producto. Conserva la calidad en productos como lechuga (Allende \& Artés, 2003). Controla el desarrollo de hongos asociados con procesos de maduración en papaya (Cia, Pascholati, Benato, Camili, \& C, 2007), uva (D'hallewin, Schirra, Pala, \& Ben-Yehoshua, 2000), zapallo (Erkan, Wang, \& Krizek, 2001).

Pero la sensibilidad de los tejidos al tratamiento con UV-C difiere en función del genotipo, y en ocasiones las dosis altas pueden favorecer la oxidación de compuestos bioactivos del fruto, como vitamina C, carotenos y fenoles así como el oscurecimiento superficial en el tejido (González-Aguilar, Villegas-Ochoa, Cuamea-Navarro, \& F, 2006; González-Aguilar, Wang, Buta, \& Krizek, 2001).
En Ecuador existen frutos exóticos como naranjilla y mortiño que son productos con alto potencial nutricional y de conocida capacidad antioxidante. Es necesario, entonces, avanzar en el estudio de la fisiología, bioquímica y aplicación de tecnologías poscosecha con el fin último de alargar el período de vida útil poscosecha y mantener o potenciar el contenido de compuestos antioxidantes y nutricionales de estos productos fruti-hortícolas. Investigaciones dirigidas con este fin permitirán planificar exportaciones de productos exóticos a destinos lejanos que por un lado demandan mayor tiempo de comercialización, y por otro lado exigen calidad del producto para que se justifique el alto valor agregado.

La naranjilla (Solanum quitoense), es una fruta climatérica, de color naranja brillante (figura 1a y 1b), de sabor y aroma exquisito, es originaria de la región interandina, específicamente de Colombia, Ecuador y Perú. En Ecuador, se destina para el mercado interno en fresco para la elaboración de jugos y pulpa. Según el Servicio de Información Agropecuaria - SICA, (2001), las variedades tradicionales de naranjilla son las de pulpa verde de jugo, que tienen el problema de la alta perecibilidad.

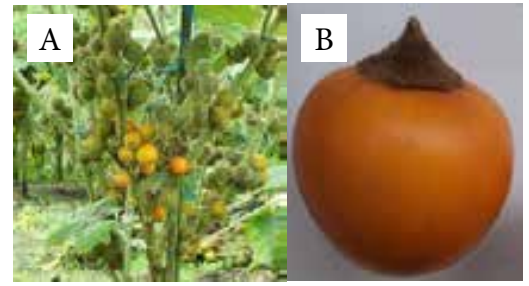

Figura 1. (A) planta; (B) fruto

La naranjilla es conocida por un alto contenido de fenoles (Acosta, Pérez, \& Vaillant, 2009; Burneo, Chamba, \& Valarezo, 2008), capacidad antioxidante (Arias, Celi, \& A, 2008) y vitamina C.

El mortiño (Vaccinium floribundum) se produce en la sierra ecuatoriana de forma silvestre (figura 2), se conoce como "Andean blueberry" y difiere del "blueberry" (arándano) que se comercializa en el mercado mundial (Servicio de Información Agropecuaria - SICA, 2001). El consumo en el Ecuador es básicamente en fresco, en un plato 
típico ecuatoriano denominado colada morada. En menor cantidad se lo utiliza para la elaboración de fruta deshidratada, mermeladas, pulpa, jugos, salsas, yogurt, fruta en conserva (especialmente para relleno en pastelería) y una variedad de confites.

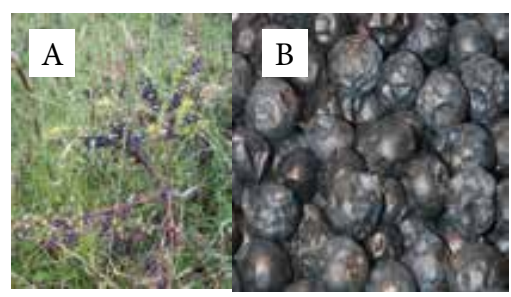

Figura 2. (A) planta; (B) fruto de mortiño

El mortiño es una buena fuente de fibra y vitamina C. Estudios realizados por Mertz et al. (2009) demuestran que el contenido de compuestos fenólicos es mayor que el encontrado en tomate de árbol y naranjilla, representando una buena fuente de compuestos antioxidantes.

El objetivo del presente estudio fue estudiar el efecto de la radiación UV-C sobre el contenido de compuestos antioxidantes y capacidad antioxidante en frutos ecuatorianos: naranjilla (Solanum quitoense) y mortiño (Vaccinium floribundum)

\section{Materiales y Métodos}

\section{Material Vegetal}

El estudio se realizó con naranjilla (Solanum quitoense) cosechada con $75 \%$ de madurez en el Cantón San Miguel de Los Bancos, provincia de Pichincha y el mortiño (Vaccinium floribundum) fue cosechado (observando su característico color azul oscuro) en el páramo El Pedregal, Cantón Mejía, Provincia de Pichincha.

Los frutos recién cosechados fueron trasladados a los laboratorios de la Universidad Tecnológica Equinoccial donde se limpiaron y clasificaron.
Los frutos se dividieron en dos grupos: control (no irradiados)y tratados (irradiados). Estos últimos se colocaron bajo cuatro lámparas UV-C (lámpara Philips, UV Germicidal G30T8) a una distancia de 30 $\mathrm{cm}$ y se irradiaron con dosis $12.5 \mathrm{~kJ} / \mathrm{m} 2$ (medida con un radiómetro digital UV (marca UVP Modelo Deluxe). Finalizado el tratamiento los frutos se colocaron en estuches de PVC tipo clamshells para el mortiño y en bandejas abiertas para la naranjilla. Las frutas tratadas se almacenaron en refrigeración $\left(5^{\circ} \mathrm{C}\right.$ ). Los controles (sin ningún tratamiento) se empacaron y almacenaron de la misma forma.

Cada 7 días, los frutos fueron retirados de la cámara de almacenamiento y se almacenaron $-20^{\circ} \mathrm{C}$ hasta su posterior análisis del contenido de compuestos antioxidantes (flavonoides, fenoles totales, ácido ascórbico, carotenoides totales, antocianinas) y capacidad antioxidante.

\section{Flavonoides (FLAV) y fenoles totales (FT)}

Se tomaron $2 \mathrm{~g}$ de tejido congelado, se trituraron y homogenizaron en $6 \mathrm{~mL}$ de etanol. La suspensión obtenida se agitó durante $60 \mathrm{~min}$, luego se separó el sobrenadante por filtración. La preparación del extracto se llevó a cabo a $4^{\circ} \mathrm{C}$. El extracto se usó para la cuantificación de FT mediante su reacción con el reactivo FolinCiocalteau según el método de (Singleton \& Rossi, 1965) y midiendo la absorbancia a $760 \mathrm{~nm}$ en un espectrofotómetro. El contenido de FLAV se evaluó mediante un ensayo espectrofotométrico según (Shin, Hai Liu, Nock, Holliday, \& Watkins, 2007) con ligeras modificaciones. A través de la formación de un complejo coloreado midiendo la absorbancia a 510nm.

\section{Ácido ascórbico (AsA)}

Fue determinado según el procedimiento descrito por Georgé, Brat, Alterar, y Amiot (2005) con ligeras modificaciones. La determinación está basada en el uso del reactivo de Folin-Ciocalteu utilizado en cuantificación de polifenoles, llevando a una estimación por diferencia.

36

Tsafiquilig=ax 


\section{Carotenoides totales}

Se tomaron $2 \mathrm{~g}$ de tejido congelado de naranjilla, se trituraron y homogenizaron en $5 \mathrm{~mL}$ de acetona durante $30 \mathrm{~min}$. Se centrifugó y conservó el sobrenadante para cuantificar los carotenoides por espectrofotometría visible. Se utilizó el método de Umiel y Gabelman (1971) las lecturas espectrofotométricas se realizaron a $503 \mathrm{~nm}$.

\section{Antocianinas}

Se tomaron $0.15 \mathrm{~g}$ de tejido liofilizado de mortiño, se trituraron y homogenizaron en $5 \mathrm{ml}$ de una solución de metanol- $\mathrm{HCl} 10 \%$ durante 30 min al abrigo de la luz. Se centrifugó y se separó el sobrenadante. Esta operación se repitió hasta obtener un pellet incoloro. Se conservó el sobrenadante para cuantificar las antocianinas por espectrofotometría según el método de Woodward (1972). Se medió la absorbancia a $515 \mathrm{~nm}$.

\section{Capacidad antioxidante}

Se prepararon los extractos el tejido según se describe para FLAV y FT; la capacidad antioxidante se evaluó por reacción con el radical estable 2,2-azino-bis (3-etilbenzotiazolin-6-ácido sulfónico) $(\mathrm{ABTS} \bullet+)$ en etanol, según el procedimiento descrito por Re et al. (1999).

\section{Análisis estadístico}

Se empleó un diseño factorial. Los resultados fueron procesados mediante un ANOVA y las medias comparadas con la prueba de diferencia mínima significativa DMS con un $\alpha=0.05$.

\section{Resultados y Discusión}

\section{Flavonoides (FLAV) y fenoles totales (FT)}

Como se presenta en la tabla 1, para la naranjilla, inmediatamente después de la aplicación de la radiación UV-C (día o) se observó menor concentración de FLAV en los frutos tratados que en los controles, el contenido de FT y AsA no se vio afectado por el tratamiento. A lo largo del almacenamiento los controles presentaron una disminución gradual alcanzando en el día 28 un 19\% menos en el contenido de FLAV que en el día inicial, mientras que los frutos tratados presentaron el mismo comportamiento desde el día 14 hasta el final del almacenamiento (día 28 ) en donde se obtiene el $23,4 \%$ menos que día o. Los frutos control presentaron mayores valores de FLAV que los frutos tratados durante el periodo de almacenamiento excepto el día 7.

\begin{tabular}{|c|c|c|c|c|}
\hline $\begin{array}{c}\text { Tiem po } \\
\text { (días) }\end{array}$ & Muestra & $\begin{array}{c}\text { FLAV } \\
\text { ( } \mu \mathrm{g} / \mathrm{g} \text { tej.) }\end{array}$ & $\begin{array}{c}F T \\
\text { ( } \mu \mathrm{g} / \mathrm{g} \text { tej.) }\end{array}$ & $\begin{array}{c}\text { AsA } \\
(\mu \mathrm{g} / \mathrm{g} \text { tej. })\end{array}$ \\
\hline \multirow{2}{*}{0} & $C$ & 363.3 & 378.8 & 472.1 \\
\hline & $\mathrm{T}$ & 352.6 & 376.1 & 456.1 \\
\hline \multirow{2}{*}{7} & C & 344.5 & 379.4 & 470.3 \\
\hline & $\mathrm{T}$ & 368.7 & 373.3 & 462.3 \\
\hline \multirow{2}{*}{14} & C & 328.4 & 365.7 & 465.1 \\
\hline & $\mathrm{T}$ & 313.7 & 368.2 & 439.7 \\
\hline \multirow{2}{*}{21} & C & 311.3 & 364.0 & 456.4 \\
\hline & $\mathrm{T}$ & 300.1 & 363.2 & 445.9 \\
\hline \multirow{2}{*}{28} & C & 293.1 & 351.9 & 431.9 \\
\hline & $\mathrm{T}$ & 269.9 & 354.2 & 369.3 \\
\hline \multicolumn{2}{|c|}{ DMS $_{a=0.05}$} & $\begin{array}{l}2.37 \\
n=6\end{array}$ & $\begin{array}{l}3.48 \\
n=6\end{array}$ & $\begin{array}{l}81.42 \\
\mathrm{n}=4\end{array}$ \\
\hline
\end{tabular}

Tabla 1. Variación del contenido de FLAV, FT y AsA en naranjilla control y tratada $(12.5 \mathrm{~kJ} / \mathrm{m2})$ durante el periodo de almacenamiento a $5^{\circ} \mathrm{C}$ *

En las naranjillas control el contenido de FT fue constante hasta el día 7, a partir de éste se observó un descenso hasta el día 28, alcanzando valores de 7,1\% menos con respecto al día o. Las naranjillas tratadas con $12.5 \mathrm{~kJ} / \mathrm{m} 2$ tuvieron un comportamiento similar a las controles alcanzando en el día 28 una concentración de 5,8\% menos que el día o. En cuanto al contenido de AsA se observó una disminución gradual del contenido en frutos controles, mientras que en frutas tratadas se mantuvo constante hasta el día 7, disminuyó en el día 14, posteriormente se presentó un incremento en el día 21 y una disminución pronunciada en el día 28. Los frutos control presentaron valores ligeramente mayores de AsA que los tratados a lo largo del almacenamiento, no obstante solamente se encontró diferencia significativa a partir del día 21. 
Por otro lado, inmediatamente después del tratamiento los frutos de mortiño presentaron mayor contenido de FLAV, FT y AsA que los controles (tabla 2). A lo largo del almacenamiento, los frutos tratados y controles presentaron reducción del contenido de FLAV, FT y AsA.

\begin{tabular}{|c|c|c|c|c|}
\hline $\begin{array}{c}\text { Tiempo de } \\
\text { almacenamiento } \\
\text { (días) }\end{array}$ & Muestra & $\begin{array}{c}\text { FLAV } \\
(\mu g / g \text { tej.) }\end{array}$ & $\begin{array}{c}F T \\
(\mu g / g \text { tej.) }\end{array}$ & $\begin{array}{c}\text { AsA } \\
(\mu g / g \text { tej. })\end{array}$ \\
\hline \multirow{2}{*}{0} & $C$ & 2.57 & 5.18 & 154 \\
\hline & $\mathrm{T}$ & 2.75 & 5.53 & 193 \\
\hline \multirow{2}{*}{7} & C & 2.28 & 6.44 & 114 \\
\hline & $\mathrm{T}$ & 2.13 & 6.27 & 163 \\
\hline \multirow{2}{*}{14} & C & 2.48 & 4.91 & 74 \\
\hline & $\mathrm{T}$ & 2.48 & 4.91 & 83 \\
\hline \multirow{2}{*}{21} & C & 2.51 & 4.34 & 47 \\
\hline & $\mathrm{T}$ & 2.64 & 4.19 & 55 \\
\hline \multirow{2}{*}{28} & C & 1.98 & 3.72 & 25 \\
\hline & $T$ & 1.76 & 3.56 & 30 \\
\hline \multicolumn{2}{|l|}{ DMS $_{a=0.05}$} & $\begin{array}{c}0.04 \\
\mathrm{n}=6\end{array}$ & $\begin{array}{c}0.14 \\
n=6\end{array}$ & $\begin{array}{l}3.83 \\
\mathrm{n}=4\end{array}$ \\
\hline
\end{tabular}

Tabla 2. Variación del contenido de FLAV, FT y AsA en mortiño control y tratado $(12.5 \mathrm{~kJ} / \mathrm{m} 2)$ durante el periodo de almacenamiento a $5^{\circ} \mathrm{C}^{*}$

En el día 7 se observó una reducción en la concentración de FLAV tanto en frutos control como en tratados. Los frutos tratados mostraron mayor concentración de FLAV con respecto a los controles en los días 14 y 21. Al final del almacenamiento (día 28) disminuyó el contenido de FLAV en un $23 \%$ en las muestras control y $36 \%$ en las muestras tratadas con respecto al día inicial (día o). Se observó un incremento del contenido de FT en el día 7 (para frutos tratados y controles), a partir del cual se produjo una disminución gradual hasta el día 28. Se encontró menor contenido de FT en las muestras tratadas en el día 21 y 28 . Al final del almacenamiento la reducción del contenido de FT fue de $35 \%$ y $28 \%$ para frutos tratados y control, respectivamente. El contenido de AsA presentó una pérdida progresiva de durante el almacenamiento, tanto para frutos tratados como controles. Hasta el día 7 los frutos tratados conservaron mayor contenido de AsA con respecto a los controles, a partir del día 14 se observó una reducción hasta el día 28 , de $53 \%$ en los control y $58 \%$ en los tratados, en relación al día inicial para las muestras tratadas y control, respectivamente.
González-Aguilar, Villegas-Ochoa, Cruz-Valenzuela, Vásquez, y Ayala-Zavala (2008) reportaron cambios en el contenido de FLAV según las condiciones de almacenamiento, al parecer estos compuestos se verían afectados por la temperatura, humedad relativa y el tiempo de almacenamiento, lo que podría explicar el comportamiento observado tanto en mortiño como en naranjilla ya que dependerá de cada sistema.

Vicente et al.(2005)y Costa, Vicente, Civello, Chaves, y Martinez (2006) reportaron que en pimiento y brócoli tratados con radiación UV-C se incrementó el contenido de FT a lo largo del almacenamiento, resultados contrarios a los encontrados en el presente estudio. La síntesis de fenoles está relacionada con la actividad enzimática de la fenilalaninaamonio liasa (PAL) (Schovánková \& Opatová, 2011), peroxidasa y polifenoloxidasa (Ciou, Lin, Chiang, Wang, \& Linton, 2011), probablemente el tratamiento UV-C afectó el metabolismo de estas enzimas de forma que no se incrementó la síntesis de fenoles en la naranjilla al igual que en mortiño.

Durante todo el periodo de almacenamiento aparentemente, no habría influencia de la radiación UV-C sobre el contenido de AsA en la naranjilla, esto podría deberse a factores como la concentración de AsA en el momento de la cosecha, regeneración de AsA debido a un proceso de biosíntesis (Jiménez, Romojaro, Gómez, Llanos, \& Sevilla, 2003) durante el almacenamiento, resistencia del fruto al estrés producido por la radiación UV-C y bajas temperaturas de almacenamiento.

\section{Carotenoides totales}

Como se muestra en la figura 3, una vez aplicada la dosis de $12.5 \mathrm{~kJ} / \mathrm{m} 2$ de radiación UV-C los frutos de naranjilla presentaron menor contenido de carotenoides que los frutos control, resultados similares fueron reportados por González-Aguilar et al. (2008) en mango fresco; posiblemente este comportamiento se deba a un tipo de estrés inducido por la luz UV-C. 


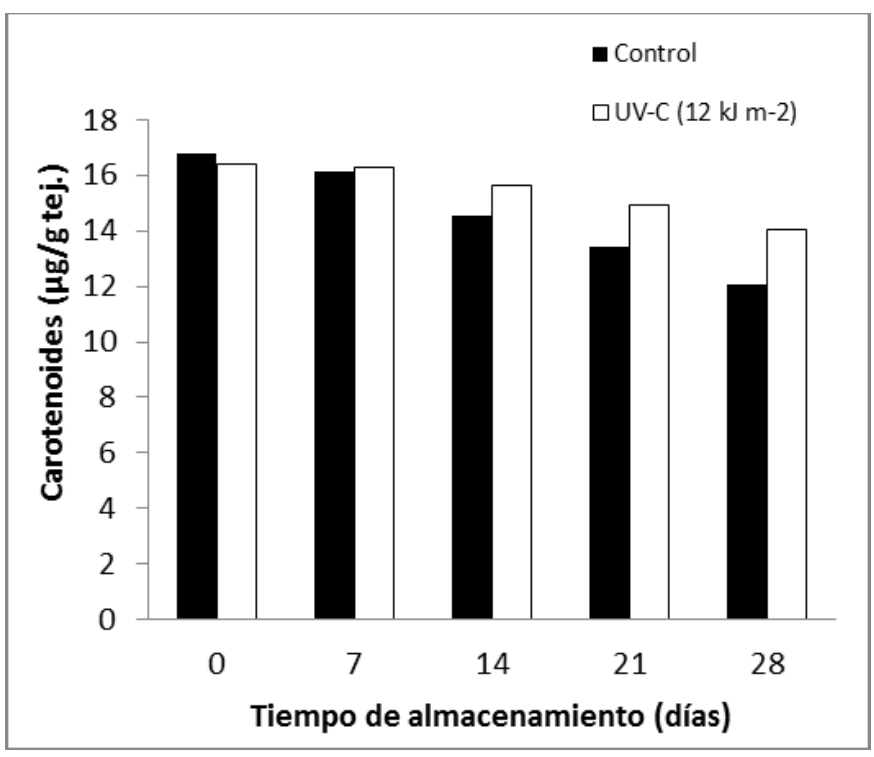

Figura 3. Contenido de carotenoides totales en naranjilla control y tratada $(12.5 \mathrm{~kJ} / \mathrm{m} 2)$ durante el almacenamiento a $5^{\circ} \mathrm{C}$. DMS. $\alpha=0.05=0.727$

Durante el almacenamiento en refrigeración en las muestras control se observó la disminución del contenido de carotenoides totales llegando en el día 28 a valores de 28,15\% menos que en el día o; las naranjillas tratadas presentaron un comportamiento similar con menor pérdida, presentando valores mayores que las muestras control, a partir del día 14 hasta el día 28 en el que tuvieron 14,5\% menos que al inicio del almacenamiento. Por acción del $\beta$-caroteno en el sistema antioxidante, la disminución encontrada podría deberse a que el estrés oxidativo del tejido se incrementa por el tratamiento con radiación UV-C. De igual manera, Vicente et al. (2005) reportaron reducción del contenido de carotenoides en pimiento irradiados y no irradiados.

\section{Antocianinas}

En el mortiño, no se observaron diferencias significativas entre las muestras control y tratadas con $12.5 \mathrm{~kJ} / \mathrm{m} 2$, inmediatamente después del tratamiento, como se observa en la figura 4. A diferencia de lo encontrado en este estudio, la aplicación de dosis de $4.3 \mathrm{~kJ} /$ $\mathrm{m} 2$ en arándano produjo un incremento en la concentración de antocianinas (Wang, Chen, \& Wang, 2009).

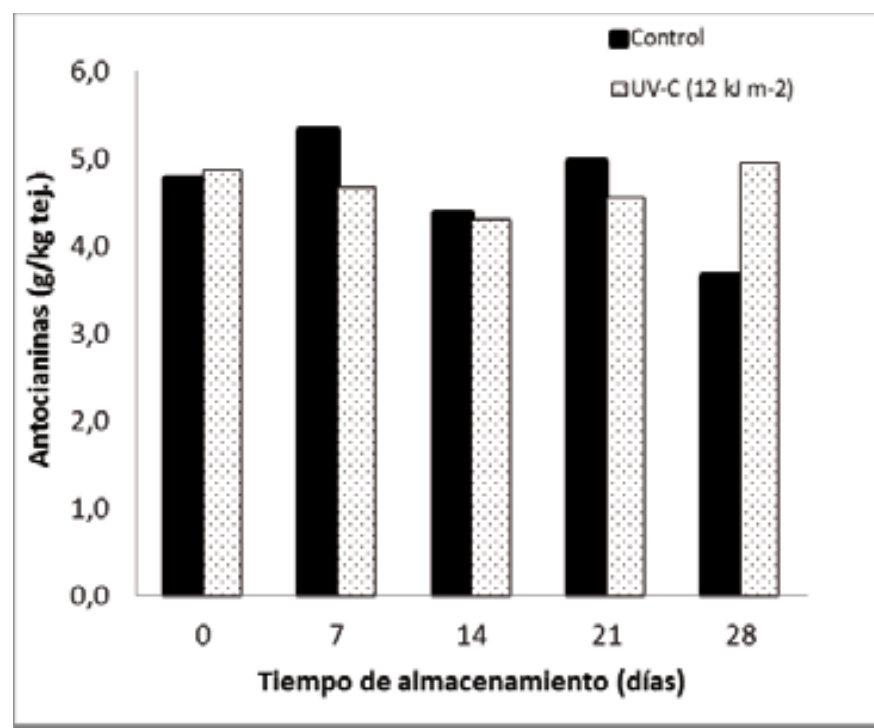

Figura 4. Contenido de antocianinas en naranjilla control y tratada $(12.5 \mathrm{~kJ} /$ $\mathrm{m} 2$ ) durante el almacenamiento a $5^{\circ} \mathrm{C}$. DMS $\alpha=0.05=0.76$

En los frutos control el contenido de antocianinas se mantuvo contante hasta el día 21 y no se encontró diferencia significativa entre los días de almacenamiento, excepto el día 28 en el cual los frutos controles presentaron una reducción del $23 \%$ con respecto al día inicial. En los frutos tratados no se encontraron diferencias significativas entre los días de almacenamiento; en el día 28 se obtuvo una concentración semejante al día o y $26 \%$ mayor concentración de antocianinas que los frutos control. Resultados similares han sido reportados por Beltrán, Ramos, y Alvares (2010) en fresas donde no se encontró cambios en el contenido de antocianinas entre frutos tratados y controles.

El tratamiento con radiación UV-C redujo la pérdida de antocianinas. En estudios realizados con arándano tratado, con radiación UV-C de 4,3 kJ/m2, el contenido de antocianinas disminuyó durante el almacenamiento (Wang et al., 2009), a diferencia de frutos como cerezas (Kataoka, Sugiyama, \& Beppu, 2003) y frutillas (Baka, Mercier, Corcuff, Castigne y Arul, 1999) donde se ha reportado que la aplicación luz UV-C favorece a la acumulación de antocianinas durante el almacenamiento. 


\section{Capacidad antioxidante}

Inmediatamente después del tratamiento con $12.5 \mathrm{~kJ} / \mathrm{m} 2$ aplicado tanto a naranjilla como a mortiño, las frutas presentaron menor capacidad antioxidante que las controles (tabla 3), por lo contrario en estudios con mangos (González-Aguilar et al., 2008), brócoli (Costa et al., 2006) y arándanos (Perkins-Veazie, Collins \& Howard, 2008) tratados con radiación UV-C en los que se observó un incremento en la capacidad antioxidante.

\begin{tabular}{|c|c|c|c|}
\hline $\begin{array}{c}\text { Tiempo de } \\
\text { almacenamiento } \\
\text { (días) }\end{array}$ & Muestra & Naranjilla & Mortiño \\
\hline \multirow{2}{*}{0} & C & 3.57 & 26.77 \\
\hline & $\mathrm{T}$ & 3.46 & 23.88 \\
\hline \multirow{2}{*}{7} & C & 3.67 & 26.92 \\
\hline & $\mathrm{T}$ & 3.75 & 23.45 \\
\hline \multirow{2}{*}{14} & C & 3.21 & 18.65 \\
\hline & $\mathrm{T}$ & 3.15 & 20.72 \\
\hline \multirow{2}{*}{21} & C & 2.55 & 16.24 \\
\hline & $\mathrm{T}$ & 2.05 & 21.55 \\
\hline \multirow{2}{*}{28} & C & 2.18 & 25.06 \\
\hline & $\mathrm{T}$ & 2.32 & 19.20 \\
\hline \multirow{2}{*}{\multicolumn{2}{|c|}{ DMS $a=0.05$}} & 0.15 & 2.85 \\
\hline & & $n=6$ & $\mathrm{n}=6$ \\
\hline
\end{tabular}

Tabla 3. Capacidad antioxidante de naranjilla y mortiño ( $\mathrm{mM}$ Trolox/g tejido), frutas control y tratadas, durante el periodo de almacenamiento a $5^{\circ} \mathrm{C}$ *

Durante el tiempo de almacenamiento de naranjillas, se observa reducción de la capacidad antioxidante, pero no se encontró diferencia significativa entre frutas control y tratadas, teniendo una pérdida total de $32 \%$ respecto al día inicial para frutos control. Mientras que la pérdida de la capacidad antioxidante de los frutos tratados fue mayor (39\%) en relación al inicio del experimento.

Para el mortiño, en los frutos control la capacidad antioxidante se mantuvo constante hasta el día 7, a partir de este día, la capacidad antioxidante disminuyó hasta el día 21 y posteriormente presentó un incremento en el día 28 con valores $6 \%$ con respecto al día inicial. La capacidad antioxidante de los frutos tratados se mantuvo constante durante 21 días de almacenamiento, en los días 14 y 21 estos frutos presentaron mayores valores que los controles; posteriormente la capacidad antioxidante de los frutos tratados se redujo en el día 28 alcanzado un 19\% menos que en el día inicial.

Tanto en naranjilla como en mortiño, a medida que aumenta el periodo de almacenamiento disminuye la capacidad antioxidante, lo que podría relacionarse con la disminución en el contenido de fenoles y flavonoides, y en menor medida a la disminución de AsA, $\beta$-caroteno (en naranjilla) y antocianinas (en mortiño) probablemente por su alta concentración en el fruto. En frutos con alta concentración de vitamina C se considera que es el componente que contribuye mayoritariamente a la capacidad antioxidante (Deepa, Kaur, Singh, \& Kapoor, 2006), sin embargo debe tomarse en cuenta el aporte que las concentraciones de $\beta$-caroteno en la naranjilla y las antocianinas en el mortiño, podría jugar un papel importante en mantener la mayor capacidad antioxidante durante períodos prolongados de almacenamiento.

\section{Conclusiones}

Inmediatamente después de la aplicación del tratamiento con radiación UV-C (12.5 kJ/m2) las naranjillas presentaron menor concentración de carotenoides y actividad antioxidante. Durante el almacenamiento prácticamente no se produjeron diferencias en el contenido de AsA, flavonoides y fenoles con respecto a las muestras control. La aplicación de la dosis de radiación UV-C (12kJ/m2) ejerció un efecto positivo principalmente sobre el contenido de carotenoides reduciendo su pérdida y prácticamente no afectó al ácido ascórbico.

Para mortiño, inmediatamente después de la aplicación de la dosis de $12,5 \mathrm{~kJ} / \mathrm{m} 2$ de radiación UV-C los frutos mostraron mayor cantidad de ácido ascórbico, fenoles totales y flavonoides. Durante el almacenamiento, el tratamiento con radiación UV-C permitió reducir las pérdidas de vitamina C (ácido ascórbico). No se produjo efecto de la radiación UV-C sobre el contenido de fenoles totales a lo largo 
del almacenamiento, tanto en frutos control como tratados; mientras que a tiempos intermedios de almacenamiento los frutos tratados presentaron mayor contenido de flavonoides que los controles. El tratamiento permitió mantener estable el contenido de antocianinas a lo largo del almacenamiento. La capacidad antioxidante de los frutos tratados $(12,5 \mathrm{~kJ} / \mathrm{m} 2)$ se mantuvo constante durante 21 días.

\section{Bibliografía}

Acosta, O, Pérez, A.M, \& Vaillant, F. (2009). Chemical characterization, antioxidant properties, and volatile constituents of naranjilla(Solanum quitoense Lam.) cultivated in Costa Rica. ALAN, 59(1), 88-94.

Allende, A, \& Artés, F. (2003). UV Radiation as a novel technique to preserve quality of fresh processed "Lollo Rosso" lettuce. Food Res. Internatl, 36, 739-746.

Arias, V, Celi, M.F, \& A, Tene. (2008). Determinación de la capacidad antioxidante en tomate de árbol (Solanum betaceam Cav.), granadilla (Pasiflora ligularis Juss.) y naranjilla (Solanum quitoense Lam.), nativas de la provincia de Loja Y Zamora Chinchipe Memorias del II Congreso Ecuatoriano de Ingeniería en Alimentos y XI Jornadas de Ciencia y Tecnología en Alimentos. Loja - Ecuador.

Artés, F, \& Allende, A. (2005). Processing lines and alternative preservation techniques to prolong the shelf-life of minimally fresh processed leafy vegetables. Eru. J. Hort. Sci, 70, 321-245.

Baka, M.; Mercier, J.; Corcuff, R.; Castaigne, F.; y Arul, J. (1999). Photochemical treatment to improve storability of fresh strawberries. Journal of Food Science, 64, 1068-1072.

Beltrán, A, Ramos, M, \& Alvares, M. (2010). Estudio de vida útil de fresa (Fragaria vesca) mediante tratamiento con radiación ultravioleta de onda corta (UV-C): Universidad Técnica de Ambato.

Burneo, I, Chamba, S, \& Valarezo, M. (2008). Determinación y Cuantificación de Compuestos Fenólicos Totales en Tomate de
Árbol (Solanum betaceam Cav.), Granadilla (Pasiflora ligularis Juss.), y Naranjilla (Solanum quitoense Lam.) Frutas Nativas de las Provincias de Loja y Zamora Chinchipe.

Cia, P, Pascholati, S.F, Benato, E.A, Camili, E.C, \& C, Santos. (2007). Effects of gamma and UV-C irradiation on the postharvest control of papaya anthracnose. Postharvest Biol. Technol, 43, 366-373.

Ciou, J, Lin, H, Chiang, P, Wang, C, \& Linton, A. (2011). The role of polyphenol oxidase and peroxidase in the browning of water caltrop pericarp during heat treatment. Food Chem, 127, 523-527.

Cisneros-Zevallos, L. (2003). The use of controlled postharvest abiotc stresses as a tool for enhancing the nutraceutical content and adding-value of fresh fruits and vegetables. J. of Food Sc, 68, 1560-1564.

Costa, L, Vicente, A. R, Civello, P. M, Chaves, A. R, \& Martinez, G. A. (2006). UV-C treatments delay postharvest senescence in broccoli florets. Postharvest Biology and Technology, 39, 204-210.

D’hallewin, G, Schirra, M, Pala, M. , \& Ben-Yehoshua, S. (2000). Ultraviolet $C$ irradiation at $0.5 \mathrm{~kJ} / \mathrm{m} 2$ reduces decay without causing damage or affecting postharvest quality of star ruby grapefruit (C. paradisi Macf.). J. Agric. Food Chem, 48, 4571-4575.

Deepa, N, Kaur, C, Singh, B, \& Kapoor, H. (2006). Antioxidant activity in some red sweet pepper cultivars. J. Food Comp. Anal(19), 572-578.

Erkan, M, Wang, C. Y, \& Krizek, D.T. (2001). UV-C irradiation reduces microbial populations and deterioration in Cucurbita pepo fruit tissue. Env. Exp. Bot, 45, 1-9.

Georgé, S, Brat, P, Alterar, P, \& Amiot, MJ. (2005). Rapid Determination of polyphenols and vitamin C in plant-derived products. Journal of Agricultural and Food Chemistry(53), 1370-1373.

González-Aguilar, G. A, Villegas-Ochoa, M. A, Cruz-Valenzuela, M. R, Vásquez, F, \& Ayala-Zavala, J. F. (2008). Irradiación (UV-C) de mango fresco cortado y su efecto en la capacidad antioxidante. Posth. Biol. And Techn, 45, 108-116. 
González-Aguilar, G. A, Villegas-Ochoa, M. A, Cuamea-Navarro, F, \& F, Ayala-Zavala J. (2006). Efecto de la irradiación UV-C sobre la calidad de mango fresco cortado. In G. A. Gónzález-Aguilar \& F. Cuamea-Navarro (Eds.), I Simposio Ibero-Americano de Vegetales Frescos Cortados (pp. 59-64).

González-Aguilar, G. A, Wang, C. Y, Buta, G. J, \& Krizek, D. T. (2001). Use of UV-C irradiation to prevent decay and maintain postharvest quality of ripe Tommy Atkins mongoes. Internatl. J. Food Sci. Technol, 36, 767-773.

Jiménez, A, Romojaro, F, Gómez, JM, Llanos, MR, \& Sevilla, F. (2003). Antioxidant systems and their relationship with the response of pepper fruits to storage at $20^{\circ} \mathrm{C}$. J. Agric. Food. Chem, 51, 6293-6299.

Kataoka, I, Sugiyama, A, \& Beppu, K. (2003). Role of ultraviolet radiation in accumulation of anthocyanin in berries of 'Gros Colman' grapes (Vitis viniferaL.). Journal of the Japanese Society for Horticultural Science, 72, 1-6.

Maharaj, R, Azul, J, \& Nadeau, P. (1999). Effect of photochemical treatment in the preservation of fres tomato (Lycopersicon esculentum cv. "(apello") by delaying senescence. Postharv. Biol. Technol, 15, 13-23.

Mercier, J, Arul, J, \& Julien, C. (1993). Effect of UV-C on phytoalexin accumulation and resistancie to Botrytis cinerea in sotred carrots. J. Phytopathol, 139, 17-35.

Mertz, C, Gancel, A. L, Gunata, Z, Alter, P, Dhuique-Mayer, C, Vaillant, F, . . Brat, P. (2009). Phenolic compounds, carotenoids and antioxidant activity of three tropical fruits. Journal of Food Composition and Analysis.

Perkins-Veazie, P., Collins, J. K. y Howard, L. (2008). Blueberry fruit response to postharvest application of ultraviolet radiation. Postharvest Biology and Technology 47, 280-285.

Re, R, Pellegrini, N, Proteggente, A, Pannala, A, Yang, M, \& Rice-Evans, C.
(1999). Antioxidant activity applying an improved ABTS radical cation decolorization assay. Biol. Med, 26, 1231-1237.

Schovánková, J, \& Opatová, H. (2011). Changes in phenols composition and activity of phenylalanine-ammonia lyase in apples after fungal infections. Technická, 5, 166128.

Servicio de Información Agropecuaria - SICA. (2001). Identificación de mercados y tecnología para productos agrícolas tradicionales de exportación. Naranjilla. CONVENIO MAG / IICA Subprograma de Cooperación Técnica (Préstamos BID / MAG 831/OC y 832/OC - EC)

Servicio de Información Agropecuaria - SICA. (2001). Identificación de mercados y tecnología para productos agrícolas tradicionales de exportación. Mortiño. CONVENIO MAG / IICA Subprograma de Cooperación Técnica (Préstamos BID / MAG 831/OC y 832/OC - EC)

Shin, Y, Hai Liu, R, Nock, J.F, Holliday, D, \& Watkins, C. (2007). Temperature and relative humidity effects on quality, total ascorbic acid, phenolics and flavonoid concentrations, and antioxidant activity of strawberry. Postharvest Biol. Technol, 45, 349-357.

Singleton, V.L, \& Rossi, Jr. J.A. (1965). Colorimetry of total phenolics with phosphomolybidic-phosphotungstic acid reagents. Am. J.Enol. Vitic, 16, 144-158.

Umiel, N, \& Gabelman, W. (1971). Analytical procedures for detecting carotenoids of carrot (Daucus carota, L.) roots and tomato (Lycopersicum esculentum) fruits. J. Am. Soc. Hortic. Sci, 96, 702- 704 .

Vicente, A, Pineda, C, Lemoine, L, Civello, P, Martinez, G, \& Chaves, A. (2005). UV-C treatments reduce decay, retain quality and alleviate chilling injury in pepper. Postharvest Biology and Technology.

Wang, C. Y, Chen, C, \& Wang, S. Y. (2009). Changes of flavonoid content and antioxidant capacity in blueberries after illumination with UV-C. Food Chemistry, 117, 426-431.

Woodward, J.R. (1972). Physical and chemical changes in developing strawberry fruits. J. Sci. Food Agric, 23, 465-473.

\section{Tsafiquiligymax}

